\title{
A New Physical Interpretation of Archimedes' Principle
}

\author{
Giancarlo Cavazzini \\ Consiglio Nazionale delle Ricerche, Istituto di Geoscienze e Georisorse, Padova, Italy \\ Email: giancarlo.cavazzini@igg.cnr.it
}

How to cite this paper: Cavazzini, G. (2018) A New Physical Interpretation of Archimedes' Principle. Journal of Applied Mathematics and Physics, 6, 215-223. https://doi.org/10.4236/jamp.2018.61020

Received: December 27, 2017

Accepted: January 26, 2018

Published: January 29, 2018

Copyright $\odot 2018$ by author and Scientific Research Publishing Inc. This work is licensed under the Creative Commons Attribution International License (CC BY 4.0).

http://creativecommons.org/licenses/by/4.0/

\begin{abstract}
The interpretation of the equilibrium of a solid body floating on the surface of a liquid body is well known as the "Archimedes' Principle". Presently, the equilibrium of the solid body is interpreted as the result of the concurrence of two mechanical actions which are equivalent and opposite: the "weight" of the body, directed downwards, and the "Archimedes' force" having a magnitude equivalent to the weight of the volume of liquid displaced by the volume of the body immersed in the liquid, directed upwards. We show arguments proving that this interpretation is not a correct physical interpretation. The same arguments show that a new different interpretation is a correct one. The new interpretation is based on the hypothesis that the "weight" of a body immersed in a body-medium is proportional to the volume of the body immersed in the body-medium and to the difference in density between the matter of the body and the matter of the body-medium. Accordingly, if a body is completely immersed in a body-medium, there is only one mechanical action on the body. This action may be downwards or upwards, or its magnitude may be zero. In this last case, the body is in equilibrium within the body-medium.
\end{abstract}

\section{Keywords}

Archimedes' Principle, Buoyancy, Density, Weight

\section{Introduction}

In the last twenty years, quite regularly, papers appeared in the literature on "Archimedes' Principle" [1]-[10]. This "Principle" [1] [2] [3] is undoubtedly the most fundamental law in hydrostatics, used to interpret a set of natural phenomena, for example "isostasy" of continental crust on the earth mantle, but it is somewhat curious, however, that physics teachers and scholars still debate about 
it approximately 2300 years after Archimedes' death.

The reasons for this discussion are fundamentally two. The first is that they are unsatisfied with the current interpretation in the cases of solid objects which sink in fluids and rest on the bottom of the respective fluid-containers. In these cases, it is difficult to explain the observed decrease in the weight of the solid body in terms of the difference in hydrostatic pressure between the bottom and the top of the body [5] [6] [7] [8] [9].

The second reason is that they feel that this "Principle" is in relation with the concept of "weight" of a body [5]-[12]. This is "inevitable", because the phenomenology studied by Archimedes introduces the concept of "density", a "property" of the matter intimately related to the "weight" of a body. Weight is one of the most important consequences of the natural process we call "gravitation", and therefore we can understand the importance of the discussion about this "Principle".

In this study, we first show the fundamental hypothesis which is at the basis of the current interpretation of the phenomenology studied by Archimedes. Then, we propose a new interpretation, which is quantitatively equivalent but not physically equivalent to the present one. Lastly, we show that the current interpretation is not a correct physical interpretation because it is in contradiction with the observations, whereas the new proposed interpretation is a correct one.

\section{The Two Hypotheses}

\subsection{The Case of a Body Completely Immersed into a Fluid}

Let us consider a solid body completely immersed in a fluid body-medium. We assume that the body-medium is a liquid body of density $d_{l i q}$. Let $V$ the volume and $d$ the density of the matter of the body. Let the body be in equilibrium within the body-medium $\left(d_{l i q}=d\right)$. The present interpretation of this phenomenology is

$$
V d=V d_{\text {liq }}
$$

i.e. the equilibrium of the body is the result of the concurrence, on the body, of two mechanical actions (called "forces") having equivalent magnitudes and opposite directions.

One of the forces is the "gravitational force" or the "weight of the body". The magnitude of this force is proportional to the volume of the body and to the density of the matter of the body. The action of this force is downwards. The second force is the "Archimedes' force" (also called "buoyancy"). The magnitude of this force is equivalent to the weight of the volume of the liquid displaced by the solid body. The action of this force is upwards.

This interpretation certainly explains the observed equilibrium. However, let us now consider it carefully. Let us consider the same body immersed not in the liquid but in the air. In this case, the body is not in equilibrium, and the present interpretation is that the mechanical disequilibrium is the result of the concur- 
rent action of two forces, the weight of the body $V d$ and the Archimedes' force $V d_{\text {air }}$

We remark that although the change in the conditions, the current interpretation assumes that the value of the weight of the body is $V d$ as in the case the body is immersed into the liquid. Whatever the body-medium, despite the differences in the physical conditions, we believe that the value of the weight of the body does not change. This is the fundamental hypothesis the present interpretation of this phenomenology is based on.

The reason for this is that we believe that the weight of a body depends on the mass of the body, i.e. on the product between volume $V$ and density $d$ of the matter of the body. Ceteris paribus, the weight of a body only depends on the mass of the body. This is the reason we have omitted gravitational acceleration $g$ in Equation (1). This view is related to the interpretation of the weight of a body in terms of an action at a distance (a "gravitational attraction" or similar) between the body and the body represented by the planet that we live on, the Earth.

However, what is the relationship between the weight of a body and the mass of the body actually? Let us consider a body in equilibrium within a fluid body-medium. In this case, we do not measure any weight of the body. If the volume of the body increases, although the mass increases, the body remains in state of equilibrium and we do not measure any increase in the weight of the body. The body remains in equilibrium as well if the volume of the body decreases: although the mass decreases, we do not measure any decrease in the weight of the body.

These arguments suggest that the relationship between the weight and the mass of a body is questionable. The process that generates the "weight" of a body seems related to the value of the density of the matter of the body rather than to the volume of the body. In particular, the process seems related to the difference in density between the matter of the body and the matter of the body-medium the body is immersed in. We measure a "weight" only if the difference in density between the matter of the body and the matter of the body-medium is not 0 .

Of course, it is possible to interpret the mechanical equilibrium of a body completely immersed in a liquid body-medium as presently (Equation (1)), but these arguments suggest that a different interpretation is also possible. Since in this case we do not measure any weight of the body, the equilibrium of the body can be interpreted as the result of the action of one single "force", which magnitude is proportional to the volume of the body and to the difference in density between the matter of the body and the matter of the fluid body-medium:

$$
V\left(d-d_{\text {liq }}\right)=0
$$

In interpretation (2), we call the expression at the first member the weight of the body in the case the body is immersed in the liquid (briefly, the weight of the body in the liquid). Ceteris paribus, the value of the weight of a body is different, depending on the body-medium the body is immersed in. The weight of a body immersed in a body-medium is proportional to the volume of the body and to 
the difference in density between the matter of the body and the matter of the body-medium. For example, the weight of the body in the case the body is immersed in the body-medium we call "(ideal) vacuum" is $V(d-0)=V d$.

We highlight that interpretation (2) is quantitatively equivalent to current interpretation (1), but the physical interpretation of the phenomenology is substantially different, because interpretation (2) assumes the existence of only one "force" acting on the body. Depending on the sign of the difference in density between the matter of the body and the matter of the body-medium, the force may be directed downwards or upwards, and in the case the difference in density is 0 , the magnitude of the force is 0 , i.e. there is no action on the body. In the last case, the body is in equilibrium within the fluid.

\subsection{The Case of a Floating Body}

The case of the equilibrium of a body completely immersed in another body is only one of the cases of the phenomenology studied by Archimedes. It is now necessary and interesting to discuss the case of a body floating on the surface of a liquid. In this case, we observe a mechanical action pushing the body upwards when we try to immerge the body completely into the liquid.

Let us consider a body of volume $V$, floating on the surface of a liquid body. Let $v$ the volume of the body immersed into the liquid. The present interpretation of the equilibrium of the body is as follows:

$$
V d=v d_{\text {liq }}
$$

i.e. the equilibrium is the result of the action on the body of two concurrent forces. One force is the "weight" of the body. The magnitude of this force is proportional to volume $V$ and to density $d$ of the matter of the body. The action of this force is downwards. The second force is the Archimedes' force. The magnitude of this force is equivalent to the weight of the volume of the liquid displaced by the volume of the body immersed in the liquid. The action of this force is upwards.

As shown in the case of Equation (2), we now do the hypothesis that the weight of a body immersed in a body-medium is proportional to the volume of the body immersed in the body-medium and to the difference in density between the matter of the body and the matter of the body-medium. In this case, a part of the body, the volume $v$, is immersed in the liquid, whereas the remaining part, the volume $V-V$, is immersed in a different body-medium. We consider the case this body-medium is the ideal vacuum $(d=0)$. Accordingly, we propose this different interpretation:

$$
(V-v)(d-0)=-v\left(d-d_{\text {liq }}\right)
$$

We read Equation (4) as follows: the equilibrium of the body is the result of the concurrent action of two forces. One force is the weight of the part (volume) of the body immersed in the vacuum. The magnitude of this force is proportional to the volume of the body immersed in this body-medium $(V-v)$, and to the 
difference in density between the matter of the body and the "matter" of the body-medium $(d-0)$. This mechanical action is downwards. The second force is the weight of the part of the body immersed into the liquid. The magnitude of this force is proportional to the volume of the body immersed in the liquid $(v)$, and to the difference in density between the matter of the body and the matter of the liquid body-medium $\left(d-d_{l i q}\right)$. This mechanical action is upwards.

Briefly: The weight of the volume of the body immersed in vacuum is equivalent and opposite in direction to the weight of the volume of the body immersed in the liquid. The negative sign at the second member in Equation (4) is necessary to obtain the numerical equivalence between the two members, because the two differences in density have different signs.

As in the case of expressions (1) and (2), we highlight that expressions (3) and (4) are quantitatively equivalent. However, they are not physically equivalent. Current interpretation (3) hypothesizes the equilibrium of the body as the result of the concurrence of forces having magnitudes higher than the magnitudes of the forces in interpretation (4).

However, exact quantitative equivalence between Equations (3) and (4) is only in the case the body-medium in which is immersed the part of the body not immersed in the liquid body-medium is the ideal vacuum. If, for example, the body-medium in which is immersed the part of the body not immersed in the liquid is the air, there is no real equivalence.

\section{Testing the Two Interpretations}

We have shown that the equilibrium of a body floating on the surface of a liquid can be interpreted in two different ways, and that in the case the body-medium in which is immersed the part of the body not immersed in the liquid is the ideal vacuum, the two hypothesis are quantitatively equivalent. Since this quantitative equivalence, one might think that it is impossible to determine whether one of the two hypotheses is wrong. This is not the case, fortunately. Let us consider the current interpretation first.

\subsection{The Current Interpretation}

Let us consider a body B floating on the surface of a liquid body. Let $B$ the volume of the body and $d, d_{\text {liq }}$ the density of the matter of the body and of the liquid body, respectively. Let us assume that a part $v$ of volume $B$ is immersed in the liquid, whereas the part $B-v=C$ is immersed in the ideal vacuum. Lastly, as an example, let us assume that $d_{\text {liq }}=3 d$, so that $V=B / 3$. This means that at equilibrium the ratio between the volume of the body immersed in the liquid and the volume of the body not immersed in the liquid is $1 / 2$. The present interpretation of the mechanical equilibrium is as follows:

$$
B d=v d_{l i q} .
$$

Let us point out the physical meaning of Equation (5): this equation means that the action of the Archimedes' force due to volume $v$ immersed in the liquid 
balances the weight of body B.

Let us now consider a second body A. Let $A$ the volume and $d$ the density of the matter of this body, and let volume $A$ such that $A=2 B$.

Let us position body $\mathrm{A}$ on the top of body $\mathrm{B}$ (as a simple example, we assume that body A and body B are two cylinders of the same radius). Since $A=2 B, B=$ $(A+B) / 3$, i.e. the new equilibrium condition is

$$
(A+B) d=(v+C) d_{l i q} .
$$

Equation (6) means that the Archimedes' force due to immersed volume $v+C$ balances the action of the weight of the whole body $\mathrm{A}+\mathrm{B}$. This means that at equilibrium no part of body $\mathrm{A}$ is immersed in the liquid.

Let us now write Equation (6) as:

$$
A d+B d=v d_{l i q}+C d_{l i q} .
$$

From balance (5) and from balance (7) follows that the buoyancy due to volume $C$ immersed in the liquid balances the weight of body A:

$$
A d=C d_{l i q} .
$$

Therefore, according to this interpretation, if we position a body of volume $A$ on the top of a body $\mathrm{C}$ of volume $C$ floating on the surface of the liquid, body $\mathrm{C}$ progressively sinks into the liquid, and as soon as it is immersed completely, the Archimedes' force balances the weight of body A. This means that at equilibrium no part of body $\mathrm{A}$ is immersed in the liquid body.

Equation (8) is not a condition of balance, however, because at equilibrium, the ratio between the volume $C$ immersed in the liquid and the volume $A+C$ of the whole body is $1 / 3$, whereas in this case, since $C=B-V=B-B / 3$ and $A=$ $2 B$, ratio $C /(A+C)$ is not $1 / 3$ but $1 / 4$. If we position a body of volume $A$ on the top of a body of volume $C$ floating on the surface of the liquid, at equilibrium a part of body A is immersed in the liquid. Therefore, the observation contradicts the current physical interpretation of the Archimedes' Principle.

\subsection{The New Interpretation}

Let us now consider the second interpretation. Equilibrium of body B is interpreted as the result of the concurrence of two equivalent and opposite actions: the weight of the part of the body immersed in the liquid (the weight of volume $v$ ) and the weight of the part of the body immersed in ideal vacuum (the weight of volume $B-v$ ). According to our quantitative definition of weight, we write

$$
(B-v)(d-0)=-v\left(d-d_{\text {liq }}\right) .
$$

i.e. the weight of the part (volume) of the body immersed in the liquid balances the weight of the part (volume) of the body immersed in ideal vacuum.

Let us now position body A on the top of body B. The physical balance is now the result of the concurrence of weight of body $\mathrm{A}$ in vacuum and weight of body $\mathrm{B}=\mathrm{v}+\mathrm{C}$ in the liquid:

$$
A(d-0)=-(v+C)\left(d-d_{l i q}\right)
$$


We write Equation (10) as:

$$
A(d-0)=-v\left(d-d_{\text {liq }}\right)-C\left(d-d_{\text {liq }}\right) .
$$

From balance (9) and from balance (11) follows the mechanical balance (volume $B-V=C$ ):

$$
A(d-0)=C(d-0)-C\left(d-d_{\text {liq }}\right)
$$

or

$$
(A-C)(d-0)=-C\left(d-d_{l i q}\right)
$$

Therefore, according to this interpretation, if we position a body of volume $A$ - $C$ on the top of a body $\mathrm{C}$ of volume $C$ floating on the surface of the liquid, body $\mathrm{C}$ progressively sinks into the liquid, and as soon as it is immersed completely, its weight balances the weight of volume $A-C$ in vacuum. This means that at equilibrium no part of body of volume $A-C$ is immersed in the liquid body.

It is easy to show that this is a real condition of equilibrium by calculating the ratio between the volume $C$ immersed in the liquid and the volume $A-C$ immersed in ideal vacuum. As we have seen in 3.1, at equilibrium this ratio is $1 / 2$. Since $C=B-v, v=B / 3$ and $A=2 B$ we have $C /(A-C)=(B-v) /(B+v)=(B-$ $B / 3) /(B+B / 3)=1 / 2$.

Again, we highlight that Equations (8) and (13) are numerically equivalent, but they are physically very different. According to current interpretation, Equation (8) is a condition of physical balance: the Archimedes' force due to the immersion of volume $C$ balances the weight of body A. This is indisputably in contradiction with the observations, however. Therefore, the interpretation of the equilibrium of a solid body floating on a liquid body as the result of the concurrent action of the weight of the body and of the Archimedes' force, equivalent to the weight of the displaced volume of liquid, is not a correct physical interpretation.

According to the interpretation we propose, Equation (13) is a condition of physical balance: the weight in the liquid of volume $C$ balances the weight in vacuum of volume $A-C$. This is in agreement with the observations. Therefore, this interpretation is a correct physical interpretation. The equilibrium of the floating body is correctly interpreted as the result of the concurrent action of two opposite equivalent "forces", each having magnitude proportional to the product between the volume of the body immersed in the body-medium and the difference in density between the matter of the body and the matter of that body-medium, respectively.

\section{Conclusions}

Presently, the equilibrium of a body floating on the surface of a liquid body-medium is interpreted as the result of the concurrent action on the body of two opposite equivalent "forces". One force is the "weight" of the body, propor- 
tional to volume and density of the matter of the body, directed downwards. The second force is the Archimedes' force, also called "buoyancy", directed upwards, equivalent to the weight of the volume of liquid displaced by the part of the body immersed in the liquid.

In this study, however, we have shown that this interpretation is not a correct physical interpretation, because it is indisputably in contradiction with the observations. We have therefore proposed a new interpretation, which is quantitatively equivalent to the current one and in agreement with the observations.

According to this interpretation, the equilibrium of the floating body is the result of the concurrent action of two opposite equivalent forces that are very different in concept and magnitude from the respective forces in the current interpretation. Each of these two forces has magnitude proportional to the product between the volume of the body immersed in a body-medium and the difference in density between the matter of the body and the matter of the body-medium, respectively.

A part $V$ of volume $V$ of the body is immersed in the liquid body-medium, whereas the remaining volume $V-V$ is immersed in a different body-medium, (the air, for example). We propose to call these two forces "weights", i.e. the weight of the volume of the body immersed in the liquid body-medium (the weight in the liquid of volume $v$ ), and the weight of the volume of the body immersed in the air (the weight in the air of volume $V-v$ ).

According to this interpretation, in the case a body is completely immersed in a fluid body-medium, only one force is acting on the body. This force may be downwards or upwards, or its magnitude may be zero. In the last case, the body is in equilibrium within the fluid body-medium.

In the case the body is completely immersed in the peculiar body-medium we call (ideal) "vacuum" $(d=0)$, the weight of the body is proportional to quantity $V(d-0)=V d$, where $d$ is the density of the matter of the body, i.e. the weight of the body is proportional to quantity we call "mass" of the body.

\section{Acknowledgements}

The author acknowledges the financial support of Consiglio Nazionale delle Ricerche, Istituto di Geoscienze e Georisorse, which made this work possible. The author is gratefully indebted to Prof. G. Borsetto for useful discussions, and declares that no conflict of interest exists with the results and conclusions presented in this paper.

\section{References}

[1] Archimedes of Siracusa. On Floating Bodies. Book I, Propositions 3-7.

[2] Halliday, D., Resnick, R. and Walker, J. (2005) Fundamentals of Physics. $7^{\text {th }}$ Edition, Wiley, New York, 367-368.

[3] Loverude, M.E., Kautz, C.H. and Heron, P.R.L. (2003) Helping Students Develop an Understanding of Archimedes' Principle. I. Research on Student Understanding. American Journal of Physics, 71, 1178-1187. https://doi.org/10.1119/1.1607335 
[4] Bierman, J. and Kincanon, E. (2003) Reconsidering Archimedes' Principle. The Physics Teacher, 41, 340-344. https://doi.org/10.1119/1.1607804

[5] Graf, E.H. (2004) Just What Did Archimedes Say about Buoyancy. The Physics Teacher, 42, 296-299. https://doi.org/10.1119/1.1737965

[6] Mohazzabi. P. (2017) Archimedes' Principle Revisited. Journal of Applied Mathematics and Physics, 5, 836-843. https://doi.org/10.4236/jamp.2017.54073.

[7] Jones, G.E. and Gordon, W.P. (1979) Removing the Buoyant Force. The Physics Teacher, 17, 59-60. https://doi.org/10.1119/1.2340127

[8] Ray, J.R. and Johnson, E. (1979) Removing the Buoyant Force, a Follow-Up. The Physics Teacher, 17, 392-393. https://doi.org/10.1119/1.2340279

[9] Taibu, R. (2017) Terms vs. Concepts-The Case of Weight. The Physics Teacher, 55, 34-35. https://doi.org/10.1119/1.4972495

[10] Taibu, R., Rudge, D. and Schuster, D. (2015) Textbook Presentations of Weight: Conceptual Difficulties and Language Ambiguities. Physical Review Physics Education Research, 11, 010117. https://doi.org/10.1103/PhysRevSTPER.11.010117

[11] Sears, F.W. (1963) Weight and Weightlessness. The Physics Teacher, 1, 20-23. https://doi.org/10.1119/1.2350557

[12] Brown, R. (1999) Weight-Don't Use the Word at All. The Physics Teacher, 37, 241-243. https://doi.org/10.1119/1.880241 